Article

\title{
Curcumin Analogues with Aldose Reductase Inhibitory Activity: Synthesis, Biological Evaluation, and Molecular Docking
}

\author{
Dasharath Kondhare ${ }^{1}$, Sushma Deshmukh ${ }^{1}$ and Harshad Lade ${ }^{2, * \mathbb{D}}$ \\ 1 School of Chemical Sciences, Swami Ramanand Teerth Marathwada University, \\ Nanded 431606, Maharashtra, India \\ 2 Department of Environmental Engineering, Konkuk University, Seoul 05029, Korea \\ * Correspondence: harshadlade@konkuk.ac.kr; Tel.: +82-2-450-3905; Fax: +82-2-450-3542
}

Received: 30 May 2019; Accepted: 25 June 2019; Published: 2 July 2019

check for updates

\begin{abstract}
Curcumin, a constituent of Curcuma longa, has shown numerous biological and pharmacological activities, including antidiabetic effects. Here, a novel series of curcumin analogues were synthesized and evaluated for in vitro inhibition of aldose reductase (AR), the first and rate-limiting enzyme of the polyol pathway, which plays a key role in the onset and progression of diabetic complications. Biological activity studies showed that all the curcuminoids exhibited moderate to good AR inhibitory (ARI) activities compared with that of the quercetin standard. Importantly, compounds $\mathbf{8 d}, \mathbf{8 h}, \mathbf{9 c}, \mathbf{9 e}$, and $\mathbf{1 0} \mathbf{g}$ demonstrated promising ARI activities, with the $50 \%$ inhibitory concentration $\left(\mathrm{IC}_{50}\right)$ values of $5.73,5.95,5.11,5.78$, and $5.10 \mu \mathrm{M}$, respectively. Four other compounds exhibited $\mathrm{IC}_{50}$ values in the range of $6.04-6.18 \mu \mathrm{M}$. Methyl and methoxy derivatives showed a remarkable ARI potential compared with that of other substitutions on the aromatic ring. Molecular docking experiments demonstrated that the most active curcuminoid (10g) was able to favorably bind in the active site of the AR enzyme. The potent ARI activities exhibited by the curcuminoids were attributed to their substitution patterns on the aromatic moiety, which may provide novel leads in the development of therapeutics for the treatment of diabetic complications.
\end{abstract}

Keywords: curcumin analogues; aldose reductase inhibitor; antidiabetic; Claisen-Schmidt condensation; molecular docking

\section{Introduction}

Diabetes mellitus (DM) is a complex metabolic disorder characterized by high blood glucose levels and the development of chronic complications, including neuropathy, nephropathy, retinopathy, and cataracts $[1,2]$. This disorder also leads to kidney failure, heart attack, and stroke, which results in more than $50 \%$ of fatalities in diabetic patients $[3,4]$. The International Diabetes Federation (IDF) has reported that an estimated 425 million adults worldwide had DM in 2017, and predicted that almost 642 million people will have diabetes by 2040 [5]. According to the IDF report, 10 countries, including three Asian countries (Indonesia, India, and Japan), will have the largest populations of diabetes patients by 2035 and will be the center of the DM epidemic [6]. Several lines of evidence have proven that $\mathrm{DM}$ is prone to chronic complications, and those are the major threat to diabetic patients [7].

Recent advances in the understanding of DM pathology have revealed that the aldose reductase (AR; alditol:(nicotinamide adenine dinucleotide phosphate (NADP+) 1-oxidoreductase, EC 1.1.1.21) enzyme activity is mainly responsible for the induction of diabetic complications. The enzyme AR is comprised of a single polypeptide chain with 315 residues. It is the first and rate-limiting enzyme in the polyol pathway of glucose metabolism, which catalyzes the reduction of glucose to sorbitol, later 
oxidized to fructose by sorbitol dehydrogenase, with NAD+ as a cofactor [4]. The substrate-binding site is in a large, deep elliptical pocket with the nicotinamide ring of aldose (NADPH) cofactor in the bottom. Even though the catalytic mechanism of AR has not yet been determined, the crystal structures reveal the active role of Tyr 48, His 110, and the nicotinamide ring in the catalytic reactions. Therefore, the inhibition of AR, which can prevent the accumulation of sorbitol, has been considered a promising therapeutic strategy for designing drugs able to delay and prevent diabetic complications.

Up to now, a wide variety of AR inhibitors, both derived from natural products and chemically synthesized, have been identified and evaluated in preclinical and clinical trials [8-10]. However, efforts to commercialize these inhibitors have been unsuccessful, as most of them were found to be clinically inadequate, owing to pharmacokinetic drawbacks, adverse side effects, or low in vivo efficacies $[9,11]$. Currently, epalrestat is the only commercially available AR inhibitory (ARI) drug, approved only in India and Japan, for the management of DM [12], but it still has some drawbacks [13,14].

Various AR inhibitors from different chemical classes appear to have certain electronic and steric characteristics in common; in particular, essential requisites for the enzyme inhibitory activity seem to be a planar structure with two hydrophobic moieties (aromatic groups) and the presence of an acidic proton, as carboxylic acid and the cyclic imide are supposed to interact with the cationic site of AR in their dissociated anionic forms [8]. Hence, the structural characteristics of phenolic compounds such as curcumin inspired us to synthesize its analogs as lead compounds containing some modifications in a number of carbons in conjugation, together with the introduction of different substitutions on aromatic groups.

It has previously been shown that curcumin [1,7-bis(4-hydroxy-3-methoxyphenyl)-1,6-heptadien3,5-dione], a diphenolic constituent found in the rhizome of the herb Curcuma longa (commonly known as turmeric), possesses multiple pharmaceutical properties, accounting for various medicinal applications, including anticarcinogenic [15,16], anti-human immunodeficiency virus (HIV)-1 [17], antibiofilm formation [18,19], anti-inflammatory [20], anti-Alzheimer's [21], anti-angiogenic [22], antimicrobial [23], antiviral [24], antimutagenic [25], antioxidant [26], and antidiabetic activities [27]. Curcumin has attracted potential therapeutic interest because it has no side effects and acts on a number of biological targets $[28,29]$. The therapeutic benefits of curcumin appear to be mainly attributable to the enolic ketone as a functional group [30] and to the presence of active methylene hydrogen [31]. However, the application of curcumin in medicine is often limited by its low bioavailability, poor aqueous solubility, and rapid metabolism [19,32]. Therefore, to overcome the limitations of the natural product curcumin and to develop potent antidiabetic agents, a series of curcumin analogs, which share similar structural features while displaying equal or better efficacies than that of curcumin, was synthesized with extended conjugation (Figure 1). As depicted in Figure 1, curcuminoids contain an extra $\mathrm{C}-\mathrm{C}$ double bond and without the moiety containing active methylene $\left(-\mathrm{CH}_{2}-\right)$ and carbonyl $(C=O)$ groups of curcumin. Hence, a chemically diverse series of molecules with a probable new mechanism of action was synthesized and evaluated for their ARI activity. The synthesis of curcuminoids was carried out by condensation of acetone and a substituted benzaldehyde or cinnamaldehyde in a base-catalyzed Claisen-Schmidt reaction. All synthesized curcuminoids were evaluated for their in vitro inhibition of an AR enzyme isolated from bovine lenses. The biological activities of the curcuminoids were further substantiated by the results of molecular docking studies. 

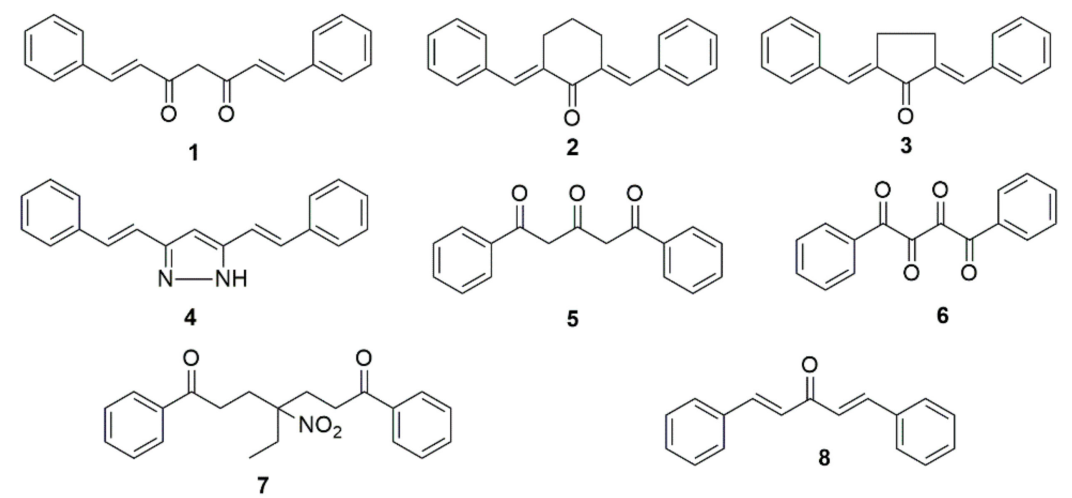

6<smiles>O=C(/C=C/C=C/c1ccccc1)/C=C/c1ccccc1</smiles><smiles>O=C(/C=C/C=C/c1ccccc1)/C=C/c1ccccc1</smiles>

Figure 1. Structures of curcumin analogues used in this study.

\section{Materials and Methods}

\subsection{Chemistry}

All starting materials and reagents were purchased from Sigma-Aldrich (St. Louis, MO, USA) and used as received. NADPH, Diethylaminoethyl (DEAE)-cellulose, DL-glyceraldehyde, ethylenediaminetetraacetic acid, and Folin-Ciocalteu's phenol reagent were procured from HiMedia Laboratories Pvt. Ltd. (Mumbai, India). Melting points of the synthesized compounds were recorded in open glass capillaries using an electrical melting point apparatus and were not corrected; $1 \mathrm{H}$ nuclear magnetic resonance (NMR) spectra were recorded on a Mercury-VX $400 \mathrm{MHz}$ NMR spectrometer (Varian, UK) at room temperature. Infrared (IR) spectra were obtained on a Nicolet iS 10 spectrometer (Thermo Fisher Scientific, Waltham, MA, USA) using $\mathrm{KBr}$ discs. Mass spectral analysis was performed on an Agilent mass spectrometer (Agilent Technology, Palo Alto, CA, USA) operated at an ionization potential of $70 \mathrm{eV}$. Reactions were monitored by thin-layer chromatography on silica gel 60 F254 plates (Merck, Darmstadt, Germany).

\subsection{Synthesis of Curcumin Analogues}

The compounds described in this study were synthesized by a straightforward chemistry according to Claisen-Schmidt condensation (Figure 2). Briefly, aldehyde (11 or 13, $2 \mathrm{mmol}$ and 13, $1 \mathrm{mmol})$ was added dropwise to a stirred solution of sodium hydroxide $(1 \mathrm{~N}, 1 \mathrm{~mL})$ and distilled ethanol $(5 \mathrm{~mL})$, and the resulting reaction mixture was stirred at the ice-cold temperature for $15 \mathrm{~min}$. Then, an appropriate ketone (12 or 14,1 mmol) was added dropwise with vigorous stirring, and the reaction mixture was allowed to stir at room temperature. After completion of the starting material, the reaction mixture was quenched by cold water $(10 \mathrm{~mL})$ and further stirred for $10 \mathrm{~min}$ at room temperature. The separated solid product was filtered, washed with cold water several times, and dried under a high vacuum. The solid was recrystallized with ethanol to afford a pure compound with a moderate to excellent yield. 
(a)<smiles>[H][R]1cccc(C(=O)[18F])c1</smiles>

(b)<smiles>[R][R]1cccc(/C=C/C(=O)[I-]C(C)=O)c1</smiles>

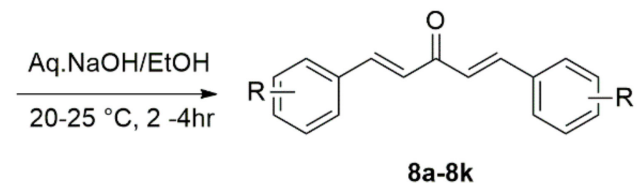

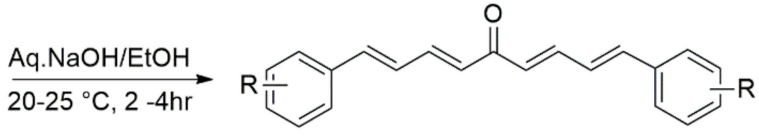

$9 a-9 i$

(c)

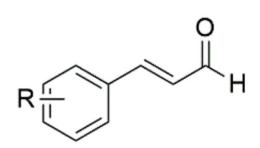

13

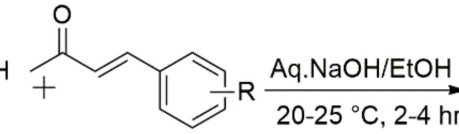

14<smiles>[R][R]1cccc(/C=C/C(=O)/C=C/C=C/c2cc#[R]cc2)c1</smiles>

10a-10i

Figure 2. Synthesis of (a) dibenzeledeneacetone, (b) dicinnamalacetone, and (c) trienonanalogs of curcumin.

2.3. Characterization of the Compounds Was Carried out Using Their Melting Point (mp), IR, $1 H$ NMR, and Mass Spectral Data as Follows

(1E,4E)-1,5-diphenylpenta-1,4-dien-3-one (8a). Pale yellow solid; mp $110-112{ }^{\circ} \mathrm{C}$. IR $\left(\mathrm{KBr}, \mathrm{cm}^{-1}\right)$ : 3025, 2941, 2932, 2848, 1650, 1622, 1613, 1535, 1460, 1363, 1220, 1120, 1063, 923, 835, 736. ${ }^{1} \mathrm{H}$ NMR $\left(400 \mathrm{MHz}, \mathrm{CDCl}_{3}\right) \delta 7.74(\mathrm{~d}, J=15.9 \mathrm{~Hz}, 2 \mathrm{H}), 7.61(\mathrm{~m}, 4 \mathrm{H}), 7.42(\mathrm{~m}, 6 \mathrm{H}), 7.11(\mathrm{~d}, J=15.9 \mathrm{~Hz}, 2 \mathrm{H}) .{ }^{13} \mathrm{C}$ NMR $\left(100 \mathrm{MHz}_{2} \mathrm{CDCl}_{3}\right) \delta 188.4,143.1,134.5,130.6,129.2,128.3,125.1$. EI MS: $m / z$ (rel. abund. \%) = $\mathrm{M}^{+1} 235$ ( $\mathrm{M}^{+}, 100 \%$ ). Anal. calcd. (\%) for $\mathrm{C}_{17} \mathrm{H}_{14} \mathrm{O}$ (234.10): C, 87.15; H, 6.02. Found: C, 87.18; H, 6.05.

(1E,4E)-1,5-bis(3,4,5-trimethoxyphenyl)penta-1,4-dien-3-one (8b). Pale yellow solid; mp $123-124{ }^{\circ} \mathrm{C}$. IR $\left(\mathrm{KBr}, \mathrm{cm}^{-1}\right)$ : 3024, 2971, 2922, 2865, 2820, 1653, 1623, 1580, 1452, 1361, 1205, 1092, 962, 748, 689. ${ }^{1} \mathrm{H}$ NMR (400 MHz, CDCl3) 87.65 (d, J = 15.9 Hz, 2H), 6.95 (d, J = 15.9 Hz, 2H), 6.82 (s, 2H), 3.93-3.95 (s, 18H). ${ }^{13} \mathrm{C}$ NMR $\left(100 \mathrm{MHz}, \mathrm{CDCl}_{3}\right) \delta 175.2,154.5,142.4,131.4,125.6,106.7,61.1,56.3$. EI MS: $\mathrm{m} / z$ (rel. abund. \%) $=\mathrm{M}^{+1}$ 415. Anal. calcd. (\%) for $\mathrm{C}_{23} \mathrm{H}_{26} \mathrm{O}_{7}$ (414.17): C, 66.65; H, 6.32. Found: C, 66.63; H, 6.30.

(1E,4E)-1,5-bis(4-bromophenyl)penta-1,4-dien-3-one (8c). Yellow solid; mp 211-213 ${ }^{\circ} \mathrm{C}$. IR $\left(\mathrm{KBr}, \mathrm{cm}^{-1}\right)$ : 3121, 2975, 2969, 2982, 2862, 2842, 1652, 1620, 1575, 1455, 1362, 1242, 1189, 955, 747, 697. ${ }^{1} \mathrm{H} \mathrm{NMR}$ $\left(400 \mathrm{MHz}, \mathrm{CDCl}_{3}\right) \delta 7.66(\mathrm{~d}, J=15.9 \mathrm{~Hz}, 2 \mathrm{H}), 7.54(\mathrm{~d}, J=8.5 \mathrm{~Hz}, 4 \mathrm{H}), 7.45(\mathrm{~d}, J=8.5 \mathrm{~Hz}, 4 \mathrm{H}), 7.05(\mathrm{~d}$, $J=15.9 \mathrm{~Hz}, 2 \mathrm{H}) .{ }^{13} \mathrm{C}$ NMR $\left(100 \mathrm{MHz}, \mathrm{CDCl}_{3}\right) \delta 143.2,140.3,134.2,133.4,130.2,126.1$. EI MS: $m / z$ (rel. abund. \%) $=\mathrm{M}^{+1}$ 391. Anal. calcd. (\%) for $\mathrm{C}_{17} \mathrm{H}_{12} \mathrm{Br}_{2} \mathrm{O}$ (389.93): C, 52.08; H, 3.08. Found: C, 52.10; H, 3.04.

(1E,4E)-1,5-bis(2,3-dimethoxyphenyl)penta-1,4-dien-3-one (8d). Yellow solid; mp 134-136 ${ }^{\circ} \mathrm{C} . \mathrm{IR}(\mathrm{KBr}$, $\left.\mathrm{cm}^{-1}\right)$ : 3327, 3125, 3067, 2975, 2932, 2846, 2810, 1648, 1628, 1571, 1466, 1363, 1205, 1180, 960, $742,690$. ${ }^{1} \mathrm{HNMR}\left(400 \mathrm{MHz}, \mathrm{CDCl}_{3}\right) \delta 8.05(\mathrm{~d}, J=16.2 \mathrm{~Hz}, 2 \mathrm{H}), 7.25(\mathrm{dd}, J=8.3,0.8 \mathrm{~Hz}, 2 \mathrm{H}), 7.15(\mathrm{~d}, J=16.2 \mathrm{~Hz}$, 2H), $7.10(\mathrm{t}, J=8.1 \mathrm{~Hz}, 2 \mathrm{H}), 6.95(\mathrm{dd}, J=8.3,0.8 \mathrm{~Hz}, 2 \mathrm{H}), 3.92(\mathrm{~s}, 6 \mathrm{H}), 3.86(\mathrm{~s}, 6 \mathrm{H}) .{ }^{13} \mathrm{C} \mathrm{NMR}(100 \mathrm{MHz}$, $\left.\mathrm{CDCl}_{3}\right) \delta 190.1,154.3,149.1,138.8,130.2,127.3,124.1,120.1,114.3,62.1,56.1$. EI MS: $\mathrm{m} / z$ (rel. abund. \%) $=\mathrm{M}^{+1}$ 355. Anal. calcd. (\%) for $\mathrm{C}_{21} \mathrm{H}_{22} \mathrm{O}_{5}$ (354.15): C, 71.17; H, 6.26. Found: C, 71.15; $\mathrm{H}, 6.25$.

(1E,4E)-1,5-bis(4-chlorophenyl)penta-1,4-dien-3-one (8e). Pale yellow solid; mp 184-186 ${ }^{\circ} \mathrm{C}$. IR $\left(\mathrm{KBr}, \mathrm{cm}^{-1}\right)$ : $3241,3125,3086,2978,2952,2927,2861,2822,1652,1620,1580,1450,1348,1214,1090,964,742,693 .{ }^{1} \mathrm{H}$ NMR (400 MHz, CDCl 3$) \delta 8.04(\mathrm{~d}, J=16.2 \mathrm{~Hz}, 2 \mathrm{H}), 7.25(\mathrm{dd}, J=8.3,0.8 \mathrm{~Hz}, 2 \mathrm{H}), 7.16(\mathrm{~d}, J=16.2 \mathrm{~Hz}$, 2H), $7.10(\mathrm{t}, \mathrm{J}=8.1 \mathrm{~Hz}, 2 \mathrm{H}), 6.95(\mathrm{dd}, J=8.3,0.8 \mathrm{~Hz}, 2 \mathrm{H}), 3.92(\mathrm{~s}, 6 \mathrm{H}), 3.90(\mathrm{~s}, 6 \mathrm{H}) .{ }^{13} \mathrm{C} \mathrm{NMR}(100 \mathrm{MHz}$, 
$\left.\mathrm{CDCl}_{3}\right) \delta 191.4,154.6,149.2,138.7,130.3,127.4,125.5,120.1,115.2,62.5,56.1$. EI MS: $m / z$ (rel. abund. \%) $=\mathrm{M}^{+1}$ 303. Anal. calcd. (\%) for $\mathrm{C}_{17} \mathrm{H}_{12} \mathrm{Cl}_{2} \mathrm{O}$ (302.03): C, 67.35; H, 3.99. Found: C, 67.33; H, 4.02.

(1E,4E)-1,5-bis(3-nitrophenyl)penta-1,4-dien-3-one (8f). Yellow solid; IR $\left(\mathrm{KBr}, \mathrm{cm}^{-1}\right)$ : 3148, 3021, 2975, 2965, 2925, 2865, 2865, 1659, 1658, 1580.3, 1455, 1362, 1275, 1095, 945, 737, 691. ${ }^{1} \mathrm{H}$ NMR $(400 \mathrm{MHz}$, $\left.\mathrm{CDCl}_{3}\right) \delta 8.52(\mathrm{~s}, 2 \mathrm{H}), 8.24(\mathrm{~d}, J=7.3 \mathrm{~Hz}, 2 \mathrm{H}), 7.92(\mathrm{~d}, J=7.5 \mathrm{~Hz}, 2 \mathrm{H}), 7.85(\mathrm{~d}, J=16.1 \mathrm{~Hz}, 2 \mathrm{H}), 7.64(\mathrm{dd}$, $J=7.9,7.9 \mathrm{~Hz}, 2 \mathrm{H}), 7.21(\mathrm{~d}, J=16.1 \mathrm{~Hz}, 2 \mathrm{H})$. EI MS: $m / z$ (rel. abund. \%) $=\mathrm{M}^{+1} 325$; Anal. calcd. $(\%)$ for $\mathrm{C}_{17} \mathrm{H}_{12} \mathrm{~N}_{2} \mathrm{O}_{5}$ (324.07): C, 62.96; H, 3.73; N, 8.64. Found: $\mathrm{C}, 62.95 ; \mathrm{H}, 3.71 ; \mathrm{N}, 8.65$.

(1E,4E)-1,5-bis(2,4-dichlorophenyl)penta-1,4-dien-3-one (8g). Pale yellow solid; IR $\left(\mathrm{KBr}, \mathrm{cm}^{-1}\right)$ : 3168, 3066, $2975,2937,2865,2818,1652,1620,1576,1452,1365,1263,1169,935,741,690 .{ }^{1} \mathrm{H}$ NMR $(400 \mathrm{MHz}$, $\left.\mathrm{CDCl}_{3}\right) \delta 7.55(\mathrm{~d}, J=15.9 \mathrm{~Hz}, 2 \mathrm{H}), 7.06(\mathrm{~d}, J=15.9 \mathrm{~Hz}, 2 \mathrm{H}), 6.62(\mathrm{~d}, J=2.2 \mathrm{~Hz}, 4 \mathrm{H}), 6.35(\mathrm{t}, J=2.2 \mathrm{~Hz}$, 2H). ${ }^{13} \mathrm{C}$ NMR $\left(100 \mathrm{MHz}, \mathrm{CDCl}_{3}\right) \delta 190.9,161.2,146.3,138.6,127.9,108.1,104.6$. EI MS: $m / z$ (rel. abund. $\%)=\mathrm{M}^{+1} 371 ; \mathrm{M}+1=372$. Anal. calcd. (\%) for $\mathrm{C}_{17} \mathrm{H}_{10} \mathrm{Cl}_{4} \mathrm{O}$ (369.95): $\mathrm{C}, 54.88 ; \mathrm{H}, 2.71$. Found: $\mathrm{C}, 54.86$; $\mathrm{H}, 2.69$.

(1E,4E)-1,5-bis-(4-methoxyphenyl)penta-1,4-dien-3-one (8h). Pale yellow solid; mp 130-132 ${ }^{\circ} \mathrm{C}$. IR (KBr, $\mathrm{cm}^{-1}$ ): 3241.4, 3162.8, 3027.3, 2978.3, 2932.5, 2862.6, 2838.4, 1654.8, 1648.8, 1578.1, 1465.6, 1363.6, 1272.2, 1075.4, 961.8, 742.4, 691.2. ${ }^{1} \mathrm{H}$ NMR $\left(400 \mathrm{MHz}, \mathrm{CDCl}_{3}\right) \delta 7.68(\mathrm{~d}, J=15.7 \mathrm{~Hz}, 2 \mathrm{H}), 7.57(\mathrm{~d}, J=8.5 \mathrm{~Hz}$, $4 \mathrm{H}), 6.94(\mathrm{~d}, J=15.7 \mathrm{~Hz}, 2 \mathrm{H}), 6.91(\mathrm{~d}, J=8.5 \mathrm{~Hz}, 4 \mathrm{H}), 3.84(\mathrm{~s}, 6 \mathrm{H}) .{ }^{13} \mathrm{C} \mathrm{NMR}\left(100 \mathrm{MHz}, \mathrm{CDCl}_{3}\right) \delta 187.8$, 162.1, 143.7, 131.2, 126.9, 124.1, 115.1, 55.8. EI MS: $m / z$ (rel. abund. \%) $=\mathrm{M}^{+1}$ 295. Anal. calcd. (\%) for $\mathrm{C}_{19} \mathrm{H}_{18} \mathrm{O}_{3}$ (294.13): $\mathrm{C}, 77.53 ; \mathrm{H}, 6.16$. Found: $\mathrm{C}, 77.55 ; \mathrm{H}, 6.14$.

(1E,4E)-1,5-di(naphthalen-2-yl)penta-1,4-dien-3-one (8i). Yellow solid; mp 244-246 ${ }^{\circ} \mathrm{C}$. IR $\left(\mathrm{KBr}, \mathrm{cm}^{-1}\right)$ : 3346.4, 3265.7, 3168.3, 3025.4, 2975.8, 2918.7, 2864.6, 2878.9, 1649.7, 1635.6, 1580.4, 1454.3, 1368.6, 1248.5, 1085.9, 961.4, 732.4, 690.1. ${ }^{1} \mathrm{H}$ NMR $\left(400 \mathrm{MHz}, \mathrm{CDCl}_{3}\right) \delta 8.04(\mathrm{~s}, 2 \mathrm{H}), 7.95(\mathrm{~d}, J=15.9 \mathrm{~Hz}, 2 \mathrm{H}), 7.83-7.86$ $(\mathrm{m}, 6 \mathrm{H}), 7.77(\mathrm{~d}, J=8.7 \mathrm{~Hz}, 2 \mathrm{H}), 7.56$ to $7.58(\mathrm{~m}, 4 \mathrm{H}), 7.26(\mathrm{~d}, J=15.9 \mathrm{~Hz}, 2 \mathrm{H})$. EI MS: $m / z$ (rel. abund. $\%)=\mathrm{M}^{+1}$ 335. Anal. calcd. (\%) for $\mathrm{C}_{25} \mathrm{H}_{18} \mathrm{O}$ (334.14): $\mathrm{C}, 89.79 ; \mathrm{H}, 5.43$. Found: $\mathrm{C}, 89.80 ; \mathrm{H}, 5.42$.

1. ,5-Bis(4-methylphenyl)-1,4-pentadien-3-one (8j). Yellow solid, $\mathrm{mp} 174-176{ }^{\circ} \mathrm{C}$. IR $\left(\mathrm{KBr}, \mathrm{cm}^{-1}\right)$ : 3246.4, $3155.7,3012.7,2970.2,2982.4,2867.8,2838.7,1643.8,1631.5,1578.1,1445.6,1367.2,1262.5,1069.9,960.8$, 741.9, 680.9. ${ }^{1} \mathrm{H}$ NMR $\left(400 \mathrm{MHz}, \mathrm{CDCl}_{3}\right) \delta 2.37(\mathrm{~s}, 6 \mathrm{H}), 7.05(\mathrm{~d}, J=15.9 \mathrm{~Hz}, 2 \mathrm{H}), 7.25(\mathrm{~m}, 4 \mathrm{H}), 7.42$ $(\mathrm{m}, 4 \mathrm{H}), 7.73(\mathrm{~d}, J=15.9 \mathrm{~Hz}, 2 \mathrm{H}) .{ }^{13} \mathrm{C} \mathrm{NMR}\left(100 \mathrm{MHz}, \mathrm{CDCl}_{3}\right) \delta 187.3,144.2,139.1,135.2,132.7,129.1$, 129.9, 126.1, 125.9, 22.4. EI MS: $m / z$ (rel. abund. $\%$ ) $=\mathrm{M}^{+1}$ 263. Anal. calcd. (\%) for $\mathrm{C}_{19} \mathrm{H}_{18} \mathrm{O}(262.14)$ : C, 86.99; H, 6.92. Found: C, 86.94; H, 6.95.

1. ,5-Bis(3-methylphenyl)-1,4-pentadien-3-one (8k). Pale yellow solid; $\mathrm{mp} 68-70{ }^{\circ} \mathrm{C}$. IR $\left(\mathrm{KBr}, \mathrm{cm}^{-1}\right)$ : 3132.6, 3086.8, 3057.6, 2978.9, 2937.4, 2866.2, 2848.7, 1645.6, 1635.7, 1578.1, 1455.3, 1362.7, 1265.2, 1146.7, 1085.4, 961.4, 741.2, 675.6. ${ }^{1} \mathrm{H}$ NMR (400 MHz, $\left.\mathrm{CDCl}_{3}\right) \delta 2.36(\mathrm{~s}, 6 \mathrm{H}), 7.05(\mathrm{~d}, J=15.9 \mathrm{~Hz}, 2 \mathrm{H}), 7.25(\mathrm{~m}, 4 \mathrm{H})$, $7.42(\mathrm{~m}, 4 \mathrm{H}), 7.71(\mathrm{~d}, J=15.9 \mathrm{~Hz}, 2 \mathrm{H}) .{ }^{13} \mathrm{C} \mathrm{NMR}\left(100 \mathrm{MHz}, \mathrm{CDCl}_{3}\right) \delta 188.3,143.7,138.3,134.4,131.2$, 128.1, 128.2, 125.3, 125.6, 21.9. EI MS: $m / z$ (rel. abund. \%) $=\mathrm{M}^{+1}$ 263. Anal. calcd. (\%) for $\mathrm{C}_{19} \mathrm{H}_{18} \mathrm{O}$ (262.14): C, 86.99; H, 6.92. Found: C, 86.93; H, 6.97.

(1E,3E,6E,8E)-1,9-diphenylnona-1,3,6,8-tetraen-5-one (9a). Yellow powder; mp $144-145^{\circ} \mathrm{C}$. IR $\left(\mathrm{KBr}, \mathrm{cm}^{-1}\right)$ : 3021, 3023.1, 2962.6, 2924.4, 2860.5, 2823.3, 1647, 1633, 1604, 1550, 1580, 1447, 1453.2, 1362.2, 1201.2, 1083.3, 966.2, 748.4, 691.2. ${ }^{1} \mathrm{H}$ NMR $\left(400 \mathrm{MHz}, \mathrm{CDCl}_{3}\right) \delta 6.58(\mathrm{~d}, J=15.2 \mathrm{~Hz}, 2 \mathrm{H}), 6.97-7.00(\mathrm{~m}, 4 \mathrm{H})$, 7.27-7.52 (m, 12H). EI MS: $m / z$ (rel. abund. \%) $=\mathrm{M}^{+1}$ 287. Anal. calcd. (\%) for $\mathrm{C}_{21} \mathrm{H}_{18} \mathrm{O}: \mathrm{C}, 88.08 ; \mathrm{H}$, 6.34. Found: C, 88.10; H, 6.35 .

(1E,3E,6E,8E)-1,9-bis(4-methoxyphenyl)nona-1,3,6,8-tetraen-5-one (9b). Yellow solid; mp 235-237 ${ }^{\circ} \mathrm{C}$. IR $\left(\mathrm{KBr}, \mathrm{cm}^{-1}\right)$ : 3010, 3018.8, 2969.7, 2918.8, 2863.1, 2829.1, 1652, 1632, 1612, 1535, 1580, 1446, 1453.6, 1359.4, 1210.5, 1092.4, 962.3, 748.6, 691.2. ${ }^{1} \mathrm{H}$ NMR $\left(400 \mathrm{MHz}, \mathrm{CDCl}_{3}\right) \delta 3.73(\mathrm{~s}, 6 \mathrm{H}), 6.56(\mathrm{~d}, J=15.2 \mathrm{~Hz}, 2 \mathrm{H})$, 6.96-7.00 (m, 4H), 7.25-7.55 (m, 10H). EI MS: $m / z$ (rel. abund. \%) $=\mathrm{M}^{+1}$ 347. Anal. calcd. (\%) for $\mathrm{C}_{23} \mathrm{H}_{22} \mathrm{O}_{3}: \mathrm{C}, 79.74 ; \mathrm{H}, 6.40$. Found: $\mathrm{C}, 79.75 ; \mathrm{H}, 6.38$. 
(1E,3E,6E,8E)-1,9-di-p-tolylnona-1,3,6,8-tetraen-5-one (9c). Yellow solid; mp 175-177 ${ }^{\circ} \mathrm{C}$. IR $\left(\mathrm{KBr}_{\mathrm{cm}}{ }^{-1}\right)$ : 3020, 3028, 2969, 2923, 2862, 2830, 1655, 1628, 1618, 1535, 1575, 1445, 1450.1, 1362.1, 1205.6, 1095.4, 960.1, 740.2, 690.5. ${ }^{1} \mathrm{H}$ NMR $\left(400 \mathrm{MHz}, \mathrm{CDCl}_{3}\right) \delta 2.33(\mathrm{~s}, 6 \mathrm{H}), 6.56(\mathrm{~d}, J=15.2 \mathrm{~Hz}, 2 \mathrm{H}), 6.95-7.00(\mathrm{~m}, 4 \mathrm{H})$, 7.25-7.51 (m, 10H). EI MS: $m / z$ (rel. abund. \%) $=\mathrm{M}^{+1}$ 315. Anal. calcd. (\%) for $\mathrm{C}_{23} \mathrm{H}_{22} \mathrm{O}: \mathrm{C}, 87.86 ; \mathrm{H}$, 7.05. Found: C, 87.84; H, 7.07.

(1E,3E,6E,8E)-1,9-bis(4-nitrophenyl)nona-1,3,6,8-tetraen-5-one (9d). Yellow solid; mp 263-266 ${ }^{\circ} \mathrm{C}$. IR ( $\mathrm{KBr}$, $\left.\mathrm{cm}^{-1}\right): 3105,3066,2975,2922,2860,2830,1763,1650,1632,1612,1542,1511,1440,1426.3,1361.4,1225.2$, 1089.3, 959.2, 715.2, 652.4. ${ }^{1} \mathrm{H}$ NMR $\left(400 \mathrm{MHz}, \mathrm{CDCl}_{3}\right) \delta 6.57(\mathrm{~d}, J=15.2 \mathrm{~Hz}, 2 \mathrm{H}), 6.96-7.05(\mathrm{~m}, 4 \mathrm{H})$, 7.23-7.50 (m, 2H), 8.04-8.13 (m, 8H). EI MS: $m / z$ (rel. abund. \%) $=\mathrm{M}^{+1} 377$. Anal. calcd. (\%) for $\mathrm{C}_{21} \mathrm{H}_{16} \mathrm{~N}_{2} \mathrm{O}_{5}$ : C, 67.02; H, 4.28; N, 7.44. Found: $\mathrm{C}, 67.05 ; \mathrm{H}, 4.30 ; \mathrm{N}, 7.45$.

(1E,3E,6E,8E)-1,9-bis(4-bromophenyl)nona-1,3,6,8-tetraen-5-one (9e). Pale yellow solid; mp 272-274 ${ }^{\circ} \mathrm{C}$. IR $\left(\mathrm{KBr}, \mathrm{cm}^{-1}\right)$ : 3226, 3122.1, 2962.2, 2910.4, 2862.3, 2817.1, 1635, 1628, 1612, 1540, 1571, 1438, 1430.1, 1337.1, 1248.1, 1090.4, 962.4, 749, 682. ${ }^{1} \mathrm{H}$ NMR $\left(400 \mathrm{MHz}, \mathrm{CDCl}_{3}\right) \delta 6.57(\mathrm{~d}, J=15.2 \mathrm{~Hz}, 2 \mathrm{H}), 6.94-7.02(\mathrm{~m}, 4 \mathrm{H})$, 7.24-7.53 (m, 10H). EI MS: $m / z$ (rel. abund. $\%$ ) $=\mathrm{M}^{+1} 444,445$. Anal. calcd. (\%) for $\mathrm{C}_{21} \mathrm{H}_{16} \mathrm{~N}_{2} \mathrm{O}_{5}: \mathrm{C}_{\text {, }}$ 56.79; H, 3.63. Found: C, 56.80; H, 3.65.

(1E,3E,6E,8E)-1,9-bis(4-(dimethylamino) phenyl)nona-1,3,6,8-tetraen-5-one (9f). Yellow solid; mp 262-264 ${ }^{\circ} \mathrm{C}$. IR $\left(\mathrm{KBr}, \mathrm{cm}^{-1}\right)$ : 3108, 3048.2, 2962.2, 2922, 2854, 2808, 1645, 1635, 1612, 1546, 1580, 1445, 1455, 1365, $1202,1080,960,743,690 .{ }^{1} \mathrm{H}$ NMR $\left(400 \mathrm{MHz}, \mathrm{CDCl}_{3}\right) \delta 3.22-3.30(\mathrm{~s}, 12 \mathrm{H}), 6.61(\mathrm{~d}, J=15.2 \mathrm{~Hz}, 2 \mathrm{H})$, 6.96-7.03 (m, 4H), 7.25-7.51 (m, 10H). EI MS: $m / z$ (rel. abund. \%) $=\mathrm{M}^{+1}$ 373. Anal. calcd. (\%) for $\mathrm{C}_{25} \mathrm{H}_{28} \mathrm{~N}_{2} \mathrm{O}$ (372.22): $\mathrm{C}, 80.61 ; \mathrm{H}, 7.58 ; \mathrm{N}$, 7.52. Found: $\mathrm{C}, 80.62 ; \mathrm{H}, 7.60 ; \mathrm{N}, 7.55$.

(1E,3E,6E,8E)-1,9-bis(4-chlorophenyl)nona-1,3,6,8-tetraen-5-one (9g). Pale yellow solid; mp 222-224 ${ }^{\circ} \mathrm{C}$. IR $\left(\mathrm{KBr}, \mathrm{cm}^{-1}\right)$ : 3010, 2970, 2922, 2861, 2833, 1649, 1635, 1625, 1545, 1530, 1443, 1453, 1361, 1205, 1109.3, 962, 742, 695. ${ }^{1} \mathrm{H}$ NMR $\left(400 \mathrm{MHz}, \mathrm{CDCl}_{3}\right) \delta 6.53(\mathrm{~d}, J=15.2 \mathrm{~Hz}, 2 \mathrm{H}), 6.96-7.01(\mathrm{~m}, 4 \mathrm{H}), 7.26-7.53(\mathrm{~m}$, 10H). EI MS: $m / z$ (rel. abund. \%) $=\mathrm{M}^{+1} 355$, 356. Anal. calcd. (\%) for $\mathrm{C}_{21} \mathrm{H}_{16} \mathrm{Cl}_{2} \mathrm{O}$ (354.06): $\mathrm{C}, 71.00 ; \mathrm{H}$, 4.54. Found: C, 71.05; H, 4.55 .

(1E,3E,6E,8E)-1,9-bis(2-nitrophenyl)nona-1,3,6,8-tetraen-5-one (9h). Yellow solid; mp 265-267 ${ }^{\circ} \mathrm{C}$. IR ( $\mathrm{KBr}$, $\left.\mathrm{cm}^{-1}\right): 3107,3077,2962,2938,2861,2825,1649,1635,1612,1540,1501,1452,1423,1364,1229,1189,961$, 743, 691. ${ }^{1} \mathrm{H}$ NMR $\left(400 \mathrm{MHz}, \mathrm{CDCl}_{3}\right) \delta 6.60(\mathrm{~d}, J=15.2 \mathrm{~Hz}, 2 \mathrm{H}), 6.94-7.01(\mathrm{~m}, 4 \mathrm{H}), 7.25-7.55(\mathrm{~m}, 6 \mathrm{H})$, 8.06-8.16 (m, 4H). EI MS: $m / z$ (rel. abund. \%) $=\mathrm{M}^{+1}$ 377. Anal. calcd. (\%) for $\mathrm{C}_{21} \mathrm{H}_{16} \mathrm{~N}_{2} \mathrm{O}_{5}: \mathrm{C}, 67.02 ; \mathrm{H}$, 4.28; N, 7.44. Found: C, 67.03; H, 4.32; N, 7.43.

(1E,3E,6E,8E)-1,9-bis(2-methoxyphenyl)nona-1,3,6,8-tetraen-5-one (9i). Yellow solid; mp 224-226 ${ }^{\circ} \mathrm{C}$. IR $\left(\mathrm{KBr}, \mathrm{cm}^{-1}\right): 3048,3017,2907,2880,2854.5,2818,1642,1631,1604,1575,1515,1470,1453,1361,1232$, $1132,945,735,695 .{ }^{1} \mathrm{H}$ NMR $\left(400 \mathrm{MHz}, \mathrm{CDCl}_{3}\right) \delta 3.63(\mathrm{~s}, 6 \mathrm{H}), 6.55(\mathrm{~d}, J=15.2 \mathrm{~Hz}, 2 \mathrm{H}), 6.96-7.02(\mathrm{~m}$, $4 \mathrm{H}), 7.27-7.52(\mathrm{~m}, 10 \mathrm{H})$. EI MS: $m / z$ (rel. abund. $\%)=\mathrm{M}^{+1} 347$. Anal. calcd. (\%) for $\mathrm{C}_{23} \mathrm{H}_{22} \mathrm{O}_{3}(346.16)$ : C, 79.74; H, 6.40. Found: C, 79.75; H, 6.38 .

(1E,4E,6E)-1,7-diphenylhepta-1,4,6-trien-3-one (10a). Yellow solid; mp 137-139 ${ }^{\circ} \mathrm{C}$. IR $\left(\mathrm{KBr}, \mathrm{cm}^{-1}\right)$ : 3348.4, 3267.9, 3148.6, 3024.7, 2970.2, 2952.6, 2865.2, 2830.3, 1643.8, 1634.7, 1607.3, 1542.4, 1575.2, 1443.7, 1450.1, 1362.1, 1246.6, 1185.5, 962.3, 742.1, 691.6. ${ }^{1} \mathrm{H}$ NMR (400 MHz, Acetone-d6) $\delta 6.62-6.64(\mathrm{~d}, J=15.2 \mathrm{~Hz}$, $2 \mathrm{H}), 6.85-6.93(\mathrm{~m}, 8 \mathrm{H}), 7.45-7.56(\mathrm{~m}, 6 \mathrm{H})$. EI MS: $\mathrm{m} / \mathrm{z}$ (rel. abund. \%) $=\mathrm{M}^{+1} 261$. Anal. calcd. (\%) for $\mathrm{C}_{19} \mathrm{H}_{16} \mathrm{O}$ (260.12): $\mathrm{C}, 87.66 ; \mathrm{H}, 6.19$. Found: $\mathrm{C}, 87.65 ; \mathrm{H}, 6.20$.

(1E,4E,6E)-7-(4-methoxyphenyl)-1-phenylhepta-1,4,6-trien-3-one (10b). Yellow solid; mp 167-169 ${ }^{\circ} \mathrm{C}$. IR $\left(\mathrm{KBr}, \mathrm{cm}^{-1}\right)$ : 3117, 3027, 2964, 2939, 2860, 2828, 1650, 1638, 1622, 1548, 1531, 1450, 1429, 1369, 1230, 1190, 960, 742, 690. ${ }^{1} \mathrm{H}$ NMR (400 MHz, Acetone-d6) $\delta 3.76(\mathrm{~s}, 3 \mathrm{H}), 6.62-6.66(\mathrm{~d}, J=15.2 \mathrm{~Hz}, 2 \mathrm{H}), 6.86-6.92$ $(\mathrm{m}, 8 \mathrm{H}), 7.42-7.7 .66(\mathrm{~m}, 5 \mathrm{H})$. EI MS: $m / z$ (rel. abund. \%) $=\mathrm{M}^{+1}$ 291. Anal. calcd. (\%) for $\mathrm{C}_{20} \mathrm{H}_{18} \mathrm{O}_{2}$ (290.13): C, 82.73; H, 6.25. Found: C, 82.71; H, 6.23. 
(1E,4E,6E)-1-phenyl-7-p-tolylhepta-1,4,6-trien-3-one (10c). Yellow solid; mp 157-159 ${ }^{\circ} \mathrm{C}$. IR $\left(\mathrm{KBr}, \mathrm{cm}^{-1}\right)$ : 3221.7, 3128.3, 2968.2, 2917.8, 2867.2, 2819.7, 1639.5, 1638.8, 1622.7, 1542.6, 1573.7, 1440.7, 1432.8, 1335.7, 1250.3, 1190.8, 965.3, 745.6, 681.6. ${ }^{1} \mathrm{H}$ NMR (400 MHz, Acetone-d6) $\delta 2.33$ (s, 3H), 6.63-6.66 (d, J = 15.2 $\mathrm{Hz}, 2 \mathrm{H}), 6.85-6.92(\mathrm{~m}, 8 \mathrm{H}), 7.42-7.65(\mathrm{~m}, 5 \mathrm{H})$. EI MS: $m / z$ (rel. abund. $\%)=\mathrm{M}^{+1}$ 275. Anal. calcd. $(\%)$ for $\mathrm{C}_{20} \mathrm{H}_{18} \mathrm{O}$ (274.14): $\mathrm{C}, 87.56 ; \mathrm{H}, 6.61$. Found: $\mathrm{C}, 87.63 ; \mathrm{H}, 6.64$.

(1E,4E,6E)-7-(4-nitrophenyl)-1-phenylhepta-1,4,6-trien-3-one (10d). Brown solid; mp 177-179 ${ }^{\circ} \mathrm{C}$. IR (KBr, $\mathrm{cm}^{-1}$ ): 3242.3, 3152.1, 3013.2, 2976.7, 2981.4, 2860.1, 2839.6, 1642.1, 1634.3, 1572.6, 1447.2, 1368.3, 1264.5, 1070.1, 962.1, 742.2, 681.6. ${ }^{1} \mathrm{H}$ NMR (400 MHz, Acetone-d6) $\delta 6.63-6.66(\mathrm{~d}, \mathrm{~J}=15.2 \mathrm{~Hz}, 2 \mathrm{H}), 6.92-7.21$ $(\mathrm{m}, 3 \mathrm{H}), 7.44-7.77(\mathrm{~m}, 6 \mathrm{H}), 8.03-8.17(\mathrm{~m}, 4 \mathrm{H})$. EI MS: $m / z$ (rel. abund. \%) $=\mathrm{M}^{+1}$ 306. Anal. calcd. $(\%)$ for $\mathrm{C}_{19} \mathrm{H}_{15} \mathrm{NO}_{3}$ (305.11): C, 74.74; $\mathrm{H}, 4.95 ; \mathrm{N}, 4.59$. Found: $\mathrm{C}, 74.72 ; \mathrm{H}, 4.96 ; \mathrm{N}, 4.60$.

(1E,4E,6E)-7-(4-bromophenyl)-1-phenylhepta-1,4,6-trien-3-one(10e). Pale yellow solid; mp 211-213 ${ }^{\circ} \mathrm{C}$. IR $\left(\mathrm{KBr}, \mathrm{cm}^{-1}\right)$ : 3245.6, 3165.6, 3025.4, 2975.2, 2932.1, 2863.1, 2839.5, 1657.1, 1652.6, 1575.6, 1462.1, 1360.1, 1271.3, 1077.2, 962.3, 740.1, 690.7. ${ }^{1} \mathrm{H}$ NMR (400 MHz, Acetone-d6) $\delta 6.60-6.64(\mathrm{~d}, \mathrm{~J}=15.2 \mathrm{~Hz}, 2 \mathrm{H})$, 6.97-7.11 (d, 2H), 7.34-7.42 (m, 3H), 7.45-7.75 (m, 8H). EI MS: $m / z$ (rel. abund. \%) $=\mathrm{M}^{+1} 339,340$. Anal. calcd. (\%) for $\mathrm{C}_{19} \mathrm{H}_{15} \mathrm{BrO}$ (338.03): C, 67.27; $\mathrm{H}, 4.46$. Found: $\mathrm{C}, 67.31 ; \mathrm{H}, 4.43$.

(1E,4E,6E)-7-(4-(dimethylamino)phenyl)-1-phenylhepta-1,4,6-trien-3-one(10f). Yellow solid; mp 267-269 ${ }^{\circ} \mathrm{C}$. IR $\left(\mathrm{KBr}, \mathrm{cm}^{-1}\right): 3348.1,3262.2,3169.8,3026.3,2970.2,2916.8,2865.1,2880.9,1659.6,1638.7,1583.6,1455.4$, 1366.1, 1247.2, 1083.1, 962.3, 734.6, 693.2. ${ }^{1} \mathrm{H}$ NMR (400 MHz, Acetone-d6) $\delta 3.04$ (s, 6H), 6.62-6.64 (d, J $=15.2 \mathrm{~Hz}, 2 \mathrm{H}), 6.96-7.13(\mathrm{~d}, 2 \mathrm{H}), 7.31-7.43(\mathrm{~m}, 3 \mathrm{H}), 7.45-7.74(\mathrm{~m}, 8 \mathrm{H})$. EI MS: $m / z$ (rel. abund. \%) = $\mathrm{M}^{+1}$ 304. Anal. calcd. (\%) for $\mathrm{C}_{21} \mathrm{H}_{21} \mathrm{NO}$ (303.16): C, 83.13; $\mathrm{H}, 6.98 ; \mathrm{N}, 4.62$. Found: $\mathrm{C}, 83.15 ; \mathrm{H}, 6.96$; $\mathrm{N}, 4.61$.

(1E,4E,6E)-7-(4-chlorophenyl)-1-phenylhepta-1,4,6-trien-3-one (10g). Pale yellow solid; mp 177-179 ${ }^{\circ} \mathrm{C}$. IR $\left(\mathrm{KBr}, \mathrm{cm}^{-1}\right)$ : 3156.7, 3068.6, 3025.4, 2970.5, 2927.6, 2862.4, 2832.8, 1658.7, 1636.7, 1612.8, 1545.7, 1571.6, 1446.4, 1426.8, 1365.9, 1270.7, 1039.9, 946.5, 748.5, 689.2. ${ }^{1} \mathrm{H}$ NMR (400 MHz, Acetone-d6) 8 6.60-6.66 (d, $\mathrm{J}=15.2 \mathrm{~Hz}, 2 \mathrm{H}), 6.97-7.12(\mathrm{~d}, 2 \mathrm{H}), 7.34-7.43(\mathrm{~m}, 3 \mathrm{H}), 7.41-7.74(\mathrm{~m}, 8 \mathrm{H})$. EI MS: $m / z$ (rel. abund. \%) = $\mathrm{M}^{+1}$ 295, 296. Anal. calcd. (\%) for $\mathrm{C}_{19} \mathrm{H}_{15} \mathrm{ClO}$ (294.08): C, 77.42; H, 5.13. Found: C, 77.44; H, 5.11 .

(1E,4E,6E)-7-(2-nitrophenyl)-1-phenylhepta-1,4,6-trien-3-one (10h). Yellow solid; mp 186-188 ${ }^{\circ} \mathrm{C}$. IR (KBr, $\left.\mathrm{cm}^{-1}\right)$ : 3104.3, 3067.4, 2972.5, 2923.6, 2862.3, 2834.2, 1765.8, 1655.2, 1634.6, 1615.7, 1545.7, 1518.3, 1445.7, 1425.2, 1360.1, 1235.9, 1069.8, 955.7, 725.8, 654.3. ${ }^{1} \mathrm{H}$ NMR (400 MHz, Acetone-d6) 8 6.62-6.63 (d, $\mathrm{J}=15.2 \mathrm{~Hz}, 2 \mathrm{H}), 6.95-7.25(\mathrm{~m}, 3 \mathrm{H}), 7.41-7.7 .71(\mathrm{~m}, 6 \mathrm{H}), 8.07-8.22(\mathrm{~m}, 4 \mathrm{H})$. EI MS: $m / z$ (rel. abund. \%) = $\mathrm{M}^{+1}$ 306. Anal. calcd. (\%) for $\mathrm{C}_{19} \mathrm{H}_{15} \mathrm{NO}_{3}$ (305.11): C, 74.74; $\mathrm{H}, 4.95 ; \mathrm{N}, 4.59$. Found: $\mathrm{C}, 74.72 ; \mathrm{H}, 4.96$; $\mathrm{N}, 4.60$.

(1E,4E,6E)-7-(2-methoxyphenyl)-1-phenylhepta-1,4,6-trien-3-one (10i). Yellow solid; mp 172-174 ${ }^{\circ} \mathrm{C}$. IR $\left(\mathrm{KBr}, \mathrm{cm}^{-1}\right)$ : 3325.7, 3127.5, 3060.7, 2972.5, 2931.2, 2843.6, 2851.6, 1645.8, 1620.8, 1575.1, 1460.6, 1365.3, 1205.5, 1184.8, 964.2, 745.1, 689.6. ${ }^{1} \mathrm{H}$ NMR (400 MHz, Acetone-d6) $\delta 3.74$ (s, 3H), 6.63-6.68 (d, J = 15.2 $\mathrm{Hz}, 2 \mathrm{H}), 6.85-6.93(\mathrm{~m}, 8 \mathrm{H}), 7.44-7.63(\mathrm{~m}, 5 \mathrm{H})$. EI MS: $\mathrm{m} / \mathrm{z}$ (rel. abund. $\%)=\mathrm{M}^{+1} 291$. Anal. calcd. $(\%)$ for $\mathrm{C}_{19} \mathrm{H}_{16} \mathrm{O}$ (290.13): C, 82.73; H, 6.25. Found: $\mathrm{C}, 82.74 ; \mathrm{H}, 6.23$.

\subsection{Determination of Bovine Lens ARI Activity of Curcuminoids}

All the synthesized curcuminoids were tested in vitro for their inhibitory activity against AR isolated from bovine lenses and purified as previously described [33]. The ARI activity of the curcuminoids was assayed spectrophotometrically (UV-1700, Shimadzu, Kyoto, Japan) by monitoring the decrease in the NADPH absorption rate at $340 \mathrm{~nm}$ over a period of $4 \mathrm{~min}$ at $30^{\circ} \mathrm{C}$, with DL-glyceraldehyde as a substrate. The reaction mixture $(900 \mu \mathrm{L})$ contained $531 \mu \mathrm{L}$ of potassium phosphate buffer (0.1 M, pH 6.2), $90 \mu \mathrm{L}$ of NADPH (1.6 mM in phosphate buffer $\mathrm{pH} 6.2), 90 \mu \mathrm{L}$ of bovine lens AR, $90 \mu \mathrm{L}$ of DL-glyceraldehyde ( $25 \mathrm{mM}$ in phosphate buffer $\mathrm{pH}$ 6.2), $90 \mu \mathrm{L}$ of deionized water, and $9 \mu \mathrm{L}$ of curcuminoids at different concentrations in dimethyl sulfoxide (DMSO). The reaction 
mixture without DL-glyceraldehyde was incubated for $10 \mathrm{~min}$ at $30^{\circ} \mathrm{C}$, and then the substrate was added to start the reaction, which was monitored for $4 \mathrm{~min}$. Quercetin, a typical ARI drug, was used as a positive control. The assay was performed in triplicate, and the inhibition percentage was calculated as follows:

$$
\text { ARI }(\%)=\left[1-\frac{(\Delta \text { A sample })-(\Delta \text { A blank })}{(\Delta A \text { control })-(\Delta \text { A blank })}\right] \times 100,
$$

where ( $\triangle \mathrm{A}$ sample) is the change in the absorbance of the reaction mixture with curcuminoids; ( $\triangle \mathrm{A}$ control) is the change in the absorbance of the reaction mixture with DMSO (without curcuminoids); and ( $\triangle \mathrm{A}$ blank) is the change in the absorbance of the reaction mixture without the AR enzyme. The results were expressed as $50 \%$ inhibitory concentration $\left(\mathrm{IC}_{50}\right)$ values, calculated from the least-squares regression line of logarithmic concentrations plotted versus the inhibitory activity.

\subsection{Molecular Docking Study}

Molecular docking studies were performed using AutoDock 4.2.6 and MGLTools 1.5.6 to investigate the intermolecular interactions between the AR enzyme and the most active curcuminoid inhibitor, $\mathbf{1 0 g}[34,35]$. The three-dimensional crystal structure of bovine AR (the enzyme we used for experiments) is not available in the Protein Data Bank (PDB) database. Hence, the crystal structure of pig AR, complexed with sorbinil (PDB: 1AH0), was used for the docking studies [36]. The structure of 1AH0 is quite similar to bovine lens AR. The MarvinSketch free software tool from the Chemaxon package was used to generate the spatial compound coordinates [37]. The receptor and ligands were prepared with the help of AutoDock tools for molecular docking simulation by adding hydrogen atoms and Kollman charges. A grid box of $60 \times 60 \times 60$ points was defined for the structure to allow the ligands to explore the possible binding site. AutoGrid 4 was used to generate grid maps for AutoDock calculations, where the search space size utilized a grid point of $1.0 \AA$. The Lamarckian genetic algorithm was used to find the best conformations of the ligand with respect to the target energy grids. Each docking experiment was performed 20 times with 250,000,000 energy evaluations, yielding 20 docked conformations. The docked conformations and the predicted model were visualized using PyMOL version 1.8.0.0 (PyMOL 1.8.0.0) [38]. The binding site for the best conformation of the ligand, with the lowest binding free energy, was analyzed.

\section{Results and Discussion}

\subsection{Chemistry}

Three different series of enone analogs of curcumin, such as (i) curcumin analogs that retained the five-carbon spacer between the aryl rings, (ii) curcumin analogs with a seven-carbon spacer, and (iii) curcumin analogs with a nine-carbon spacer, were synthesized to evaluate their ARI activity. Scanned ${ }^{1} \mathrm{H}$ NMR and IR spectral data of synthesized compounds are given in the Supplementary Materials. All the synthesized curcumin analogs contain two identical aryl rings separated by an unsaturated five-carbon spacer with a single carbonyl, whereas some compounds have two different aryl rings (Table 1). These curcuminoids were designed to investigate the effect of the length of the spacer between the two aryl rings. The desired derivatives were obtained in $70 \%-85 \%$ yield after purification. Curcuminoids are known for a poor solubility; however, owing to the extended conjugation between the two aromatic moieties, a good aqueous solubility was observed for the synthesized curcuminoids. Additionally, it was found that the introduction of halogen groups increased the solubility of these curcuminoids in both protic and aprotic solvents such as methanol-water dioxane and DMSO. Therefore, the results obtained in this study would be useful for designing and synthesizing curcuminoids with enhanced aqueous solubility. 
Table 1. Synthesis of a series of curcumin analogues.

\begin{tabular}{|c|c|c|c|c|c|c|c|}
\hline Entry & Product & $\begin{array}{l}\text { Time } \\
\text { (hr.) }\end{array}$ & $\begin{array}{l}\text { Yield } \\
(\%)\end{array}$ & Entry & Product & $\begin{array}{l}\text { Time } \\
\text { (hr.) }\end{array}$ & $\begin{array}{c}\text { Yield } \\
(\%)\end{array}$ \\
\hline $8 a$ & & 2 & 85 & $9 e$ & & 2 & 84 \\
\hline $8 b$ & & 2 & 84 & 9f & & 4 & 70 \\
\hline $8 c$ & & 2.5 & 85 & $9 \mathrm{~g}$ & & 2 & 82 \\
\hline $8 d$ & & 2 & 85 & $9 h$ & & 3.5 & 75 \\
\hline $8 e$ & & 2 & 82 & $9 \mathrm{i}$ & & 3 & 74 \\
\hline $8 f$ & & 2.5 & 80 & $10 a$ & & 2 & 85 \\
\hline $8 \mathrm{~g}$ & & 2 & 84 & $10 \mathrm{~b}$ & & 2 & 84 \\
\hline $8 \mathrm{~h}$ & & 2.5 & 85 & $10 \mathrm{c}$ & & 3 & 82 \\
\hline $8 \mathrm{i}$ & & 2 & 84 & $10 \mathrm{~d}$ & & 2 & 84 \\
\hline $8 \mathrm{j}$ & & 2.5 & 82 & $10 \mathrm{e}$ & & 2 & 85 \\
\hline $8 k$ & & 3 & 80 & $10 \mathrm{f}$ & & 3.5 & 80 \\
\hline $9 a$ & & 2 & 80 & $10 \mathrm{~g}$ & & 2 & 84 \\
\hline $9 b$ & & 2 & 78 & $10 \mathrm{~h}$ & & 3 & 81 \\
\hline $9 \mathrm{c}$ & & 2.5 & 78 & $10 \mathrm{i}$ & & 3 & 80 \\
\hline $9 d$ & & 3.5 & 80 & & & & \\
\hline
\end{tabular}

\subsection{AR Inhibition Study}

AR is best characterized as a glucose-reducing NAD $(\mathrm{P}) \mathrm{H}$-dependent oxidoreductase enzyme responsible for the pathophysiology of DM disorders [39]. Therefore, the ARI activity of the synthesized curcuminoids was evaluated by measuring the reduction of DL-glyceraldehyde in the presence of NADPH as a reductant. The results showed that all curcuminoids of the novel series displayed a good to moderate activity against AR at micromolar concentrations (Table 2), when compared with that of the known standard quercetin. The compounds tested showed AR inhibition levels 
from $53.1 \% \pm 0.9 \%$ to $75.4 \% \pm 1.9 \%$ at the $10 \mu \mathrm{M}$ concentration. Compounds $8 \mathbf{d}, \mathbf{8 h}, \mathbf{9 c}, \mathbf{9 e}$, and $\mathbf{1 0} \mathbf{g}$ showed promising ARI activities, with $\mathrm{IC}_{50}$ values of $5.73 \pm 0.28,5.95 \pm 0.27,5.11 \pm 0.11,5.78 \pm 0.13$, and $5.10 \pm 0.14 \mu \mathrm{M}$, respectively. Furthermore, compounds $\mathbf{8 a}, \mathbf{8 i}, \mathbf{8 j}$, and $\mathbf{9 g}$ showed AR inhibition levels from $56.8 \% \pm 1.2 \%$ to $68.7 \% \pm 1.8 \%$ at the $10 \mu \mathrm{M}$ concentration, with $\mathrm{IC}_{50}$ values of $6.04 \pm 0.32$, $6.12 \pm 0.16,6.08 \pm 0.14$, and $6.18 \pm 0.29 \mu \mathrm{M}$, respectively. The dose response plot for compounds $8 \mathrm{a}$, $\mathbf{8 b}, \mathbf{9 a}, \mathbf{1 0 a}$, and 10b with standard quercetin is given in the Supplementary Materials (Figure S1). These results suggested that the insertion of the spacer between the two rings allowed a more extensive electronic delocalization, which supports the affinity of the inhibitors to AR. It was also observed that methyl and methoxy derivatives of curcumin (e.g., 8b, 8d, 8h, 8j, 8k, 9b, 9c, 9e, 9i, 10b, 10c, and 10i) exhibited more significant ARI activities than those shown by compounds with other substitutions on the aromatic ring. This indicated that the substitution pattern on the aromatic moiety affected the ARI activity of the derivatives. Additionally, the synthesized curcuminoids might possess the antioxidant activity, as it has been previously reported that methoxy groups are involved in antioxidant properties $[40,41]$. Previously, curcumin analogs were synthesized and evaluated for inhibitory effects on bovine lens AR activity $[40,41]$. The result of the structure-activity relationship demonstrated that curcumin analogs possessing the ortho-dihydroxyl group formed a close affinity with AR to exhibit potent inhibitory effects.

Table 2. Inhibitory effects of the curcumin analogues on bovine lens aldose reductase (AR).

\begin{tabular}{|c|c|c|c|c|c|}
\hline Entry & Inhibition (\%) ${ }^{a}$ & $\mathrm{IC}_{50}(\mu \mathrm{M})^{\mathrm{b}}$ & Entry & Inhibition (\%) ${ }^{a}$ & $\mathrm{IC}_{50}(\mu \mathrm{M})^{\mathrm{b}}$ \\
\hline $8 a$ & $66.3 \pm 1.3$ & $6.04 \pm 0.32$ & $9 e$ & $75.4 \pm 1.9$ & $5.78 \pm 0.13$ \\
\hline $8 b$ & $64.4 \pm 1.2$ & $6.83 \pm 0.27$ & $9 f$ & $66.7 \pm 1.6$ & $6.65 \pm 0.31$ \\
\hline $8 c$ & $61.7 \pm 1.1$ & $6.23 \pm 0.21$ & $9 \mathrm{~g}$ & $68.7 \pm 1.8$ & $6.18 \pm 0.29$ \\
\hline $8 d$ & $74.4 \pm 1.6$ & $5.73 \pm 0.28$ & $9 h$ & $65.4 \pm 1.5$ & $6.72 \pm 0.26$ \\
\hline $8 \mathrm{e}$ & $64.2 \pm 1.2$ & $6.80 \pm 0.24$ & $9 \mathrm{i}$ & $63.6 \pm 1.3$ & $7.00 \pm 0.30$ \\
\hline $8 f$ & $68.4 \pm 1.4$ & $6.23 \pm 0.18$ & $10 a$ & $67.5 \pm 1.7$ & $6.45 \pm 0.21$ \\
\hline $8 g$ & $67.5 \pm 1.5$ & $6.62 \pm 0.33$ & $10 \mathrm{~b}$ & $58.7 \pm 1.0$ & $6.54 \pm 0.19$ \\
\hline $8 \mathrm{~h}$ & $63.5 \pm 1.3$ & $5.95 \pm 0.27$ & $10 \mathrm{c}$ & $62.4 \pm 1.1$ & $6.80 \pm 0.16$ \\
\hline $8 \mathrm{i}$ & $66.5 \pm 1.8$ & $6.12 \pm 0.16$ & $10 \mathrm{~d}$ & $54.1 \pm 0.9$ & $8.01 \pm 0.32$ \\
\hline $8 \mathbf{j}$ & $56.8 \pm 1.2$ & $6.08 \pm 0.14$ & $10 \mathrm{e}$ & $65.7 \pm 1.5$ & $6.57 \pm 0.21$ \\
\hline $8 k$ & $64.4 \pm 1.4$ & $6.78 \pm 0.23$ & $10 f$ & $71.4 \pm 1.7$ & $5.85 \pm 0.16$ \\
\hline $9 a$ & $53.1 \pm 0.9$ & $9.02 \pm 0.28$ & $10 \mathrm{~g}$ & $68.6 \pm 1.6$ & $5.10 \pm 0.14$ \\
\hline $9 b$ & $58.8 \pm 1.0$ & $10.65 \pm 0.34$ & $10 \mathrm{~h}$ & $67.3 \pm 1.6$ & $6.62 \pm 0.29$ \\
\hline $9 c$ & $67.8 \pm 1.7$ & $5.11 \pm 0.11$ & $10 \mathrm{i}$ & $64.6 \pm 1.4$ & $6.70 \pm 0.15$ \\
\hline $9 d$ & $66.7 \pm 1.6$ & $6.64 \pm 0.15$ & Quercetin & $81.5 \pm 2.2$ & $5.01 \pm 0.09$ \\
\hline
\end{tabular}

Data are expressed as the means \pm standard deviation $(n=3)$. ${ }^{\text {a }}$ The AR inhibitory (ARI) activity was determined at a concentration of $10 \mu \mathrm{M} .{ }^{\mathrm{b}}$ The $50 \%$ inhibitory concentration $\left(\mathrm{IC}_{50}\right)$ values represent the concentration required to decrease the AR activity by $50 \%$, as estimated from the least-squares regression line of logarithmic concentrations $(10,25,50$, and $100 \mu \mathrm{M})$ vs. \% inhibition. Quercetin was used as a positive control.

It was also observed that halogen-containing curcuminoids (e.g., $8 c, 8 g, 9 e, 9 g, 10 e$, and $10 \mathrm{~g}$ ) exhibited an excellent ARI activity, with $\mathrm{IC}_{50}$ values ranging from $5.10 \pm 0.14$ to $6.62 \pm 0.33 \mu \mathrm{M}$. The $\alpha, \beta$-double bond is known to decrease ARI activity, while the increase in conjugation promotes the inhibition of AR. It was noted that most of the curcumin analogs with ARI activity retained the phenolic ring substituents. However, a number of the analogs lacking phenolic ring substituents, which were capable of forming stable tertiary carbon-centered radicals, were also active. Similarly, several researchers have tried to enhance the therapeutic properties and stability of curcumin analogs through structural modifications [42,43]. Thus, the observed ARI activity of the curcuminoids is therapeutically important because the low bioavailability of curcumin limits its medicinal application. The results of biological evaluation were further confirmed by the molecular docking studies. 


\subsection{Molecular Docking}

Molecular docking analysis is vital for the design of drugs as it not only highlights the correspondence between the biological activity and binding, but also elucidates the key interactions with the active site. In order to gain some insight about the binding mode of the designed inhibitors, the docking analysis of synthesized curcumin analogues in the active site of pig AR (PDB: 1AH0) were carried out. Herein, to further understand the intermolecular interactions between the most active curcuminoid $(\mathbf{1 0 g})$ and the AR enzyme, molecular docking studies were performed using AutoDock 4.2.6 and MGLTools 1.5.6. Figure 3 shows the interacting mode of curcuminoid $10 \mathrm{~g}$ in the binding site of AR. As shown in Figure 3, the potent ARI curcuminoid $10 \mathrm{~g}\left(\mathrm{IC}_{50}=5.10 \mu \mathrm{M}\right)$ fits well into the active site of the AR enzyme.

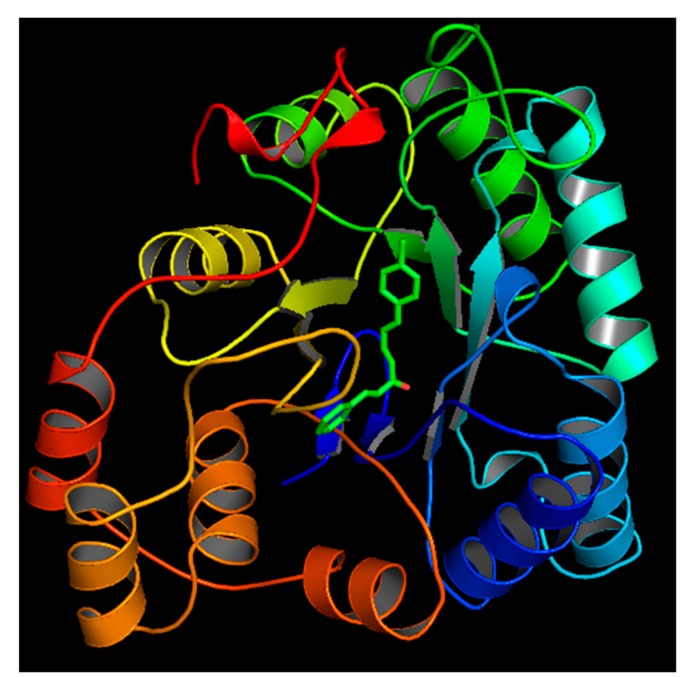

Figure 3. Docking view of curcuminoid 10g (molecular weight $=294.77$ ) (green sticks) in the binding site of AR. The protein structures are shown in ribbon representation.

Curcuminoid $10 \mathrm{~g}$ demonstrated a prominent steric interaction with AR through the formation of a hydrogen bond between $\mathrm{CO}$ and NH of the amino acids Trp20 and Thr19 (Figure 4).

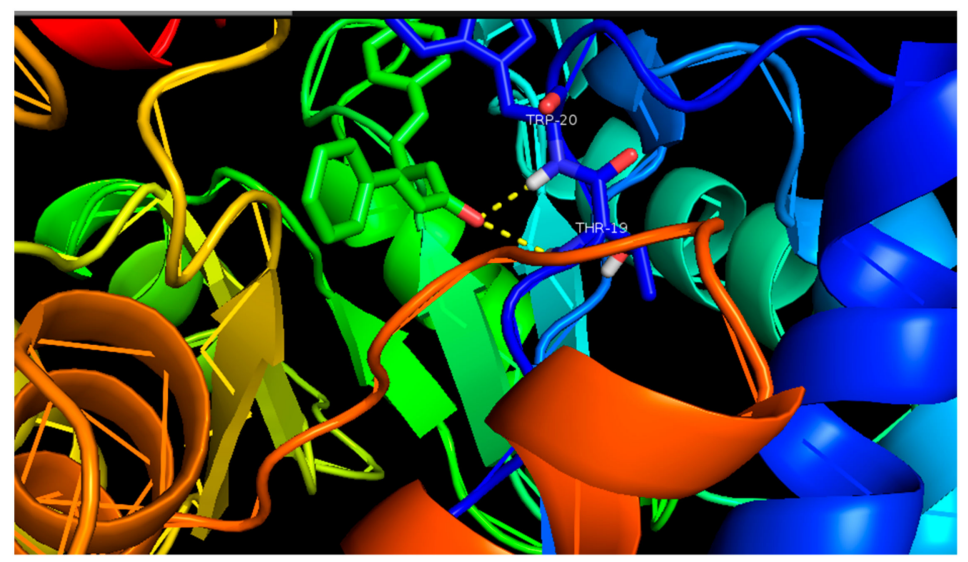

Figure 4. Ligand-receptor interaction mode of curcuminoid $10 \mathrm{~g}$ with the AR enzyme. The interaction of $\mathbf{1 0 g}$ between oxygen (red) and hydrogen (grey) of amino acids.

It was observed that the binding pocket could accommodate curcuminoids with various substituents, which allows further modifications to increase the potency. 


\section{Conclusions}

A novel series of curcumin analogs was designed, synthesized, and evaluated in vitro for the inhibition of AR. The biological activity study revealed that all the synthesized curcuminoids exhibited moderate to good ARI activity, which indicated the successful synthesis of AR inhibitors. Importantly, the halogen-containing curcuminoids exhibited excellent ARI activity, thereby providing important evidence for further development of antidiabetic drugs. Molecular docking studies performed on the most active curcumin analog provided a deeper insight into the binding mode of the synthesized curcuminoids, thus creating a basis for further development of potent and selective AR inhibitors to treat diabetes and associated complications.

Supplementary Materials: The following are available online at http://www.mdpi.com/2227-9717/7/7/417/s1. Scanned 1H NMR and IR spectral data of important synthesized compounds and Figure S1. Dose response plot for compounds $8 \mathrm{a}, 8 \mathrm{~b}, 9 \mathrm{a}, 10 \mathrm{a}$ and $10 \mathrm{~b}$ with standard quercetin.

Author Contributions: Conceptualization, H.L. and D.K.; Methodology, D.K. and S.D.; Validation, D.K. and H.L.; Formal analysis, D.K. and S.D.; Investigation, D.K. and S.D.; Data curation, D.K.; Writing一original draft preparation, H.L. and D.K.; Writing—review and editing, H.L.; Visualization, H.L. and D.K.; Supervision, H.L.

Funding: This research received no external funding.

Acknowledgments: This research was supported by the KU Research Professor Program of Konkuk University.

Conflicts of Interest: The authors declare no conflict of interest.

\section{References}

1. Brownlee, M. Biochemistry and molecular cell biology of diabetic complications. Nature 2001, 414, 813-820. [CrossRef] [PubMed]

2. Alexiou, P.; Pegklidou, K.; Chatzopoulou, M.; Nicolaou, I.; Demopoulos, V.J. Aldose reductase enzyme and its implication to major health problems of the 21st century. Curr. Med. Chem. 2009, 16, 734-752. [CrossRef] [PubMed]

3. World Health Organization. Global Report on Diabetes. Available online: http://www.who.int/diabetes/ globalreport/en/ (accessed on 22 November 2017).

4. Kador, P.F. The role of aldose reductase in the development of diabetic complications. Med. Res. Rev. 1988, 8, 325-352. [CrossRef] [PubMed]

5. International Diabetes Federation. IDF Diabetes Atlas-8th Edition. 2017. Available online: http://www. diabetesatlas.org/ (accessed on 10 May 2019).

6. Whiting, D.R.; Guariguata, L.; Weil, C.; Shaw, J. IDF diabetes atlas: Global estimates of the prevalence of diabetes for 2011 and 2030. Diabetes Res. Clin. Pract. 2011, 94, 311-321. [CrossRef] [PubMed]

7. Ramana, K.V.; Srivastava, S.K. Aldose reductase: A novel therapeutic target for inflammatory pathologies. Int. J. Biochem. Cell Biol. 2010, 42, 17-20. [CrossRef]

8. Kador, P.J.; Kinoshita, J.H.; Sharpless, N.E. Aldose reductase inhibitors: A potential new class of agents for the pharmacological control of certain diabetic complications. J. Med. Chem. 1985, 28, 841-849. [CrossRef]

9. Costantino, L.; Rastelli, G.; Cignarella, G.; Vianello, P.; Barlocco, D. New aldose reductase inhibitors as potential agents for the prevention of long-term diabetic complications. Exp. Opin. Ther. Pat. 1997, 7, 843-858. [CrossRef]

10. Costantino, L.; Rastelli, G.; Gamberini, M.C.; Barlocco, D. Pharmacological Approaches to the Treatment of Diabetic Complications. Exp. Opin. Ther. Pat. 2000, 10, 1245-1262. [CrossRef]

11. Changjin, Z. Aldose reductase inhibitors as potential therapeutic drugs of diabetic complications. In Diabetes Mellitus_Insights and Perspectives; Oguntibeju, O.O., Ed.; InTech: Rijeka, Croatia, 2013; pp. 17-46.

12. Maccari, R.; Ciurleo, R.; Giglio, M.; Cappiello, M.; Moschini, R.; Corso, A.D.; Mura, U.; Ottanà, R. Identification of new non-carboxylic acid containing inhibitors of aldose reductase. Bioorg. Med. Chem. 2010, 18, 4049-4055. [CrossRef]

13. Ramirez, M.A.; Borja, N.L. Epalrestat: An aldose reductase inhibitor for the treatment of diabetic neuropathy. Pharmacotherapy 2008, 28, 646-655. [CrossRef] 
14. Sharma, S.R.; Sharma, N. Epalrestat, an aldose reductase inhibitor, in diabetic neuropathy: An Indian perspective. Ann. Indian Acad. Neurol. 2008, 11, 231-235. [CrossRef] [PubMed]

15. Abdel-Lateef, E.; Mahmoud, F.; Hammam,O.; El-Ahwany, E.; El-Wakil, E.; Kandil, S.; Taleb, H.A.; El-Sayed, M.; Hassenein, H. Bioactive chemical constituents of Curcuma longa L. rhizomes extract inhibit the growth of human hepatoma cell line (HepG2). Acta Pharm. 2016, 66, 387-398. [PubMed]

16. Basile, V.; Ferrari, E.; Lazzari, S.; Belluti, S.; Pignedoli, F.; Imbriano, C. Curcumin derivatives: Molecular basis of their anti-cancer activity. Biochem. Pharmacol. 2009, 78, 1305-1315. [CrossRef] [PubMed]

17. Ali, A.; Banerjea, A.C. Curcumin inhibits HIV-1 by promoting Tat protein degradation. Sci. Rep. 2016, 6, 27539. [CrossRef] [PubMed]

18. Lade, H.; Song, W.J.; Yu, Y.J.; Ryu, J.H.; Arthanareeswaran, G.; Kweon, J.H. Exploring the potential of curcumin for control of $\mathrm{N}$-acyl homoserine lactone-mediated biofouling in membrane bioreactors for wastewater treatment. RSC Adv. 2017, 27, 16392-16400. [CrossRef]

19. Packiavathy, I.A.; Priya, S.; Pandian, S.K.; Ravi, A.V. Inhibition of biofilm development of uropathogens by curcumin-An anti-quorum sensing agent from Curcuma longa. Food Chem. 2014, 148, 453-460. [CrossRef] [PubMed]

20. Kohli, K.; Ali, J.; Ansari, M.J.; Raheman, Z. Curcumin: A natural anti-inflammatory agent. Indian J. Prarmacol. 2005, 37, 141-147. [CrossRef]

21. Mishra, S.; Palanivelu, K. The effect of curcumin (turmeric) on Alzheimer's disease: An overview. Ann. Indian Acad. Neurol. 2008, 11, 13-19. [CrossRef]

22. Folkman, J. Can mosaic tumor vessels facilitate molecular diagnosis of cancer? Proc. Natl. Acad. Sci. USA 2001, 98, 398-400. [CrossRef]

23. Mun, S.H.; Joung, D.K.; Kim, Y.S.; Kang, O.H.; Kim, S.B.; Seo, Y.S.; Kim, Y.C.; Lee, D.S.; Shin, D.W.; Kweon, K.T.; et al. Synergistic antibacterial effect of curcumin against methicillin-resistant Staphylococcus aureus. Phytomedicine 2013, 20, 714-718. [CrossRef]

24. Hergenhahn, M.; Soto, U.; Weninger, A.; Polack, A.; Hsu, C.H.; Cheng, A.L.; Rösl, F. The chemopreventive compound curcumin is an efficient inhibitor of Epstein-Barr virus BZLF1 transcription in Raji DR-LUC cells. Mol. Carcinog. 2002, 33, 137-145. [CrossRef] [PubMed]

25. Shukla, Y.; Arora, A.; Taneja, P. Anti-mutagenic potential of curcumin on chromosomal aberrations in Wistarrats. Mutat. Res. 2002, 515, 197-202. [CrossRef]

26. Braga, M.E.M.; Leal, P.F.; Carvalho, J.E.; Meireles, M.A.A. Comparison of yield, composition, and antioxidant activity of turmeric (Curcuma longa L.) extract obtained using various techniques. J. Agric. Food Chem. 2003, 51, 6604-6611. [CrossRef] [PubMed]

27. Arun, N.; Nalini, N. Efficacy of turmeric on blood sugar and polyol pathway in diabetic albino rats. Plant Foods Hum. Nutr. 2002, 57, 41-52. [CrossRef] [PubMed]

28. Siviero, A.; Gallo, E.; Maggini, V.; Gori, L.; Mugelli, A.; Firenzuoli, F.; Vannacci, A. Curcumin, a golden spice with a low bioavailability. J. Herb. Med. 2015, 5, 57-70. [CrossRef]

29. Mahmood, K.; Zia, K.M.; Zuber, M.; Salman, M.; Anjum, M.N. Recent developments in curcumin and curcumin based polymeric materials for biomedical applications: A review. Int. J. Biol. Macromol. 2015, 81, 877-890. [CrossRef]

30. Priyadarsini, K.I. Chemical and structural features influencing the biological activity of curcumin. Curr. Pharm. Des. 2013, 19, 2093-2100.

31. Priyadarsini, K.I.; Maity, D.K.; Naik, G.H.; Kumar, M.S.; Unnikrishnan, M.K.; Satav, J.G.; Mohan, H. Role of phenolic $\mathrm{O}-\mathrm{H}$ and methylene hydrogen on the free radical reactions and antioxidant activity of curcumin. Free Radic. Biol. Med. 2003, 35, 475-484. [CrossRef]

32. Rudrappa, T.; Bais, H.P. Curcumin, a known phenolic from Curcuma longa, attenuates the virulence of Pseudomonas aeruginosa PAO1 in whole plant and animal pathogenicity models. J. Agric. Food Chem. 2008, 56, 955-962. [CrossRef]

33. Hayman, S.; Kinoshita, J.H. Isolation and properties of lens aldose reductase. J. Biol. Chem. 1965, 240, 877-882.

34. Morris, G.M.; Huey, R.; Lindstrom, W.; Sanner, M.F.; Belew, R.K.; Goodsell, D.S.; Olson, A.J. AutoDock4 and AutoDockTools4: Automated docking with selective receptor flexibility. J. Comput. Chem. 2009, 30, 2785-2791. [CrossRef] [PubMed] 
35. Sanner, M.F. Python: A programming language for software integration and development. J. Mol. Graph. Model. 1999, 17, 57-61. [PubMed]

36. Urzhumtsev, A.; Tête-Favier, F.; Mitschler, A.; Barbanton, J.; Barth, P.; Urzhumtseva, L.; Biellmann, J.F.; Podjarny, A.; Moras, D. A 'specificity' pocket inferred from the crystal structures of the complexes of aldose reductase with the pharmaceutically important inhibitors tolrestat and sorbinil. Structure 1997, 5, 601-612. [CrossRef]

37. Csizmadia, P. MarvinSketch and MarvinView: Molecule applets for the World Wide Web. In Proceedings of the 3rd International Electronic Conference on Synthetic Organic Chemistry (ECSOC-3), Basel, Switzerland, 1-30 September 1999; Sciforum Electronic Conference Series. Volume 3, pp. 367-369.

38. The PyMOL Molecular Graphics System; Version 1.8.0.0; Schrödinger, LLC: New York, NY, USA, 2015.

39. Schrijvers, B.F.; de Vriese, A.S.; Flyvbjerg, A. From hyperglycemia to diabetic kidney disease: The role of metabolic, hemodynamic, intracellular factors and growth factors/cytokines. Endocr. Rev. 2004, 25, 971-1010. [CrossRef] [PubMed]

40. Somparn, P.; Phisalaphong, C.; Nakornchai, S.; Unchern, S.; Morales, N.P. Comparative antioxidant activities of curcumin and its demethoxy and hydrogenated derivatives. Biol. Pharm. Bull. 2007, 30, 74-78. [CrossRef] [PubMed]

41. Du, Z.Y.; Bao, Y.D.; Liu, Z.; Qiao, W.; Ma, L.; Huang, Z.S.; Gu, L.Q.; Chan, A.S. Curcumin analogs as potent aldose reductase inhibitors. Arch. Pharm. Weinh. 2006, 339, 123-128. [CrossRef] [PubMed]

42. Ferrari, E.; Pignedoli, F.; Imbriano, C.; Marverti, G.; Basile, V.; Venturi, E.; Saladini, M. Newly synthesized curcumin derivatives: Crosstalk between chemico-physical properties and biological activity. J. Med. Chem. 2011, 54, 8066-8077. [CrossRef] [PubMed]

43. Lee, K.H.; Ab Aziz, F.H.; Syahida, A.; Abas, F.; Shaari, K.; Israf, D.A.; Lajis, N.H. Synthesis and biological evaluation of curcumin-like diarylpentanoidanalogues for anti-inflammatory, antioxidant and anti-tyrosinase activities. Eur. J. Med. Chem. 2009, 44, 3195-3200. [CrossRef] [PubMed]

(C) 2019 by the authors. Licensee MDPI, Basel, Switzerland. This article is an open access article distributed under the terms and conditions of the Creative Commons Attribution (CC BY) license (http://creativecommons.org/licenses/by/4.0/). 